\title{
Odor intensity and pleasantness for a diverse set of odorants
}

\author{
HOWARD R. MOSKOWITZ \\ Food Science Laboratory, U.S. Army Natick Laboratories, Natick, Massachusetts 01760 \\ ANDREW DRAVNIEKS \\ Odor Sciences Center, Mlinois Institute of Technology Research Institute, Chicago, Ilinois 60645
}

and

LEON A. KLARMAN

Data Analysis Office, U.S. Army Natick Laboratories, Natick, Massachusetts 01760

\begin{abstract}
Observers compared the odor of butanol to the odors of various concentrations of 32 chemicals, and also rated the odor pleasantness and unpleasantness of these 32 odorants by a line matching procedure. All stimuli were presented to the observers by air dilution olfactometers. Odor intensity matches between each odorant and butanol were often describable by power functions. A derived magnitude estimate of odor intensity was obtained through a calibration of the butanol concentration scale. Most odorants grew less rapidly in intensity than butanol, and all odorants grew in intensity as decelerating functions of concentration. A more general function of the form $P=k_{1}+k_{2} C^{k 3}$ was needed to account for the pleasantness-unpleasantness judgments. In this study, most odorants were rated either neutral or unpleasant. Observers showed far more variability in hedonic judgments than in intensity judgments.
\end{abstract}

The present paper concerns relations between odor concentration, odor intensity, and odor pleasantness. Although a variety of studies have been published which concern one odorant or a few selected odorants, those studies investigated odor intensity only, and not the relation between odor intensity and odor pleasantness (see Berglund, Berglund, Engen, \& Ekman, 1971, and Cain \& Moskowitz, 1974, for a tabulation of such experiments in olfaction). A second aim of the present study was to investigate two procedures (equal intensity odor matching and line production for hedonics) which can be of use in field situations where the methods of numerical ratio and magnitude estimation may not be easily implemented.

The quantification of olfactory hedonics, pleasantness and unpleasantness of odor impressions, poses a challenging problem to both the scientist working in a laboratory setting with odorants of known chemical purity and to the applied researcher faced with measuring and eliminating noxious odorants. Odorants may vary in pleasantness with concentration, and odorants tend to evoke mental images and recapture past experiences, adding still further a cognitive input with its own pleasant and unpleasant associations. In a study of 1-butanol in the laboratory setting, observers showed greater agreement in their judgments of odor intensity than of odor pleasantness. Some observers found 1-butanol to become increasingly pleasant with concentration, although

Requests for reprints should be sent to Howard R. Moskowitz, MPI Sensory Testing. Inc., 770 Lexington Avenue, New York, New York 10021 . most observers reported just the opposite (Moskowitz, Dravnieks, \& Gerbers, 1974). Quite often, the naive subject responds primarily to the hedonic component of an odor, before considering its quality and intensity (Yoshida, 1964).

Traditional assessments of odor hedonics have been made with either category scales or with unidimensional ratio scales. The 9-point "hedonic scale" of preference (Peryam \& Pilgrim, 1957) is often used to assess a variety of hedonic aspects of stimuli, including aroma (odor). This scale allows for four categories reflecting graded degrees of liking, four reflecting graded degrees of disliking, and a category for neutrality: Ratio scales, erected by magnitude estimation, usually have not allowed this distinction-observers faced with unpleasant odors were instructed to assign to those odors low numbers (Engen \& McBurney, 1964; Henion, 1971). The present study uses a bipolar scale for hedonics, adapted from both the hedonic scale and from the method of cross-modal matching. Observers are instructed first to label whether they like an odor, dislike it, or find it neutral (an example of bipolar scales), and then they are instructed to draw a line as long as they feel represents the strength of their affective response (an example of cross-modal matching). Line-length matching produces functions for intensity almost equivalent to functions produced by magnitude estimation. Line-length matching can also be used with children, and has been used to test the affective responses of illiterate Indian laborers to varied taste materials (Moskowitz, Sharma, Jacobs, Sharma, \& Kumraiah, 1976) with success. 
intensities, from below threshold to extremely strong. The Observer altempted to match the unknown stimulus odorant to one of the eight butanol levels, by a forced-choice matching procedure. The matching level of butanol was recorded in parts per million in air. The check run, butanol vs. butanol, produced almost $100 \%$ correspondence between butanol as an unknown vapor and the matching level of butanol.

When assessing pleasantness or unpleasantness, the observer was instructed to draw a line that was as long (subjectively) as the odorant was pleasant or unpleasant. This was accompanied by a statement as to whether the odor was pleasant or unpleasant. This two-step procedure is a variety of cross-modality matching, in which line length substitutes for numerical estimation. Both line length and number are related by a power function, whose exponent is 1.0. so that a 10 -fold increase in the length of line implies a 10 -fold increase in the jud ged pleasantness (or unpleasantness) as obtained by magnitude estimation. Since ach observer drew lines according to his personal scake unit (modulus), the results were normalized prior to averaging the results from different observers. The observer was instructed to draw a line that represented "moderate." All line lengths for each observer were then divided by this length to provide commensurate noduli across observers.

\section{Analysis}

The direct eyual-intensity matches between each stimulus and butanol (across concentrations) were expressed in logarithmic values (log percentage dilution of the odorant in air vs. $\log$ PPM matching butanol). The logarithms of the matching levels of butanol were then averaged to yield a mean matehing tevel of butanol. If the function relating these two logarithmic values turns out to be a straight line in log-log coordinates, then the equal-intensity matching function can be represented by a power function: $B-k^{n}(B=$ matching level of butanol; $T=$ concentration of test odorant-e.g., percent dilution; $k$ and $n$ are the intercept and the exponent, respectively).

The empirical matching equation above, expressed in terms of butanol level, as well as the standardized intensity function proposed by Moskuwit\%, Dravnieks, Cain, and Turk (1974), may be combined to related each test odorant to a derived magnitude estimate:

$$
M=0.261(k)^{0.66}(\mathrm{~T})^{0.66 \mathrm{n}}
$$

According to Equation 1, one needs to determine only two parameters for the butanol matching function. The remaining parameters can then be calculated by multiplication and exponentiation.

Analysis of the pleasantness functions is much more difficult, owing to the positive and negative values that are possible when judging liking vs. disliking. A simple power function of the form shown in Equation 1 is not feasible, since it does not cross the zero level to become negative. On the other hand, a moditication of the power function may be made, in which negative numbers are permissible. The modification produces the slightly more complicated function:

$$
P=k_{1}+k_{2} C^{k_{3}}
$$

Eyuation 2 presents a power function with an additive constant. If $k_{1}$ is positive and $k_{2}$ is positive, then the pleasantness judgments can never be less than 0 . The variation of signs for $k_{1}$ and $k_{2}$ allows for various points at which the odorant becomes neutral and unpleasant with concentration. The more complicated term was chosen to continue representing hedonic function by power iunctions.

Simple power functions are assigned slopes (exponents) and intercepts (multipliers), based upon least squares estimates. Analytical solutions for both the exponent and the intercept exist, since the power functions can be linearized by double logarithmic transformation. For Equation 2, an analytic solution is not possible. Therefore, iterature solutions must be obtained in hopes of obtaining a minimum-residual solution. The pleasantness functions of the present study were fitted by a series of least squares lines for various values of $k_{3}$ (Equation 2). As $k_{3}$ was systematically varied over a wide range of values (between +11 and 11 ), each exponent produced a set of $k_{1}$ and $k_{2}$ values, as well as a residual sum of squares. That value of $k_{3}$ minimizing the residual sum of squares was selected as the optimum solution.

\section{RESULTS}

\section{Intensity Functions}

Figure 1 presents the various intensity functions, shown as equal-intensity matching functions against butanol. Although many of the functions approximate straight lines in $\log -\log$ coordinates, a variety of odorants either level off at the top concentrations or at the bottom concentrations. As a first approximation, therefore, only a subset of all odorants in the present study can really be considered to grow as power functions when sensory intensity is being judged. Those flattening out at top concentrations may have been too strong to be easily matched by butanol.

The important finding of this part of the study is that most equal-intensity matching functions are governed by slopes (in log-log coordinates) that are lower than 1.0 against butanol odor intensity. Therefore, butanol grows more rapidly in perceived intensity than the majority of other odorants. If the butanol transformation function is used to obtain derived magnitude estimates, then all of these matching slopes (lower than 1.0) should be multiplied by 0.66 to obtain an estimate of power function exponent (see Table 1). Hence, according to these results, virtually every single odorant grows as a decelerating function of concentration. Tenfold increases in concentration almost never produce tenfold increases in odor intensity, no matter what odorant is being evaluated. In contrast, for taste, some chemicals produce intensity power functions that are accelerating functions of concentration (e.g., the sweetness of glucose; Moskowitz, 1971). In this respect, the two chemical senses differ quite dramatically from each other, since, for olfaction, in virtually every experiment reported that deals with the direct scaling of odor intensity decelerating functions are found.

The suggestion that air dilution of odorants produces higher power functions than corresponding liquid dilution of the same odorants (Cain, 1969) is affirmed in the present study. Although no liquid dilutions were used, the absolute values of the exponents are higher here than in studies with dilutions in liquid solvents (e.g., paraffin oil, diethyl pthalate, benzyl benzoate; see Berglund et al., 1971; Cain, 1969; Cain \& Moskowitz, 1974). The reason for this difference is not altogether clear, although physical factors involved in liquid dilution may play a role in providing a smaller actual range of 

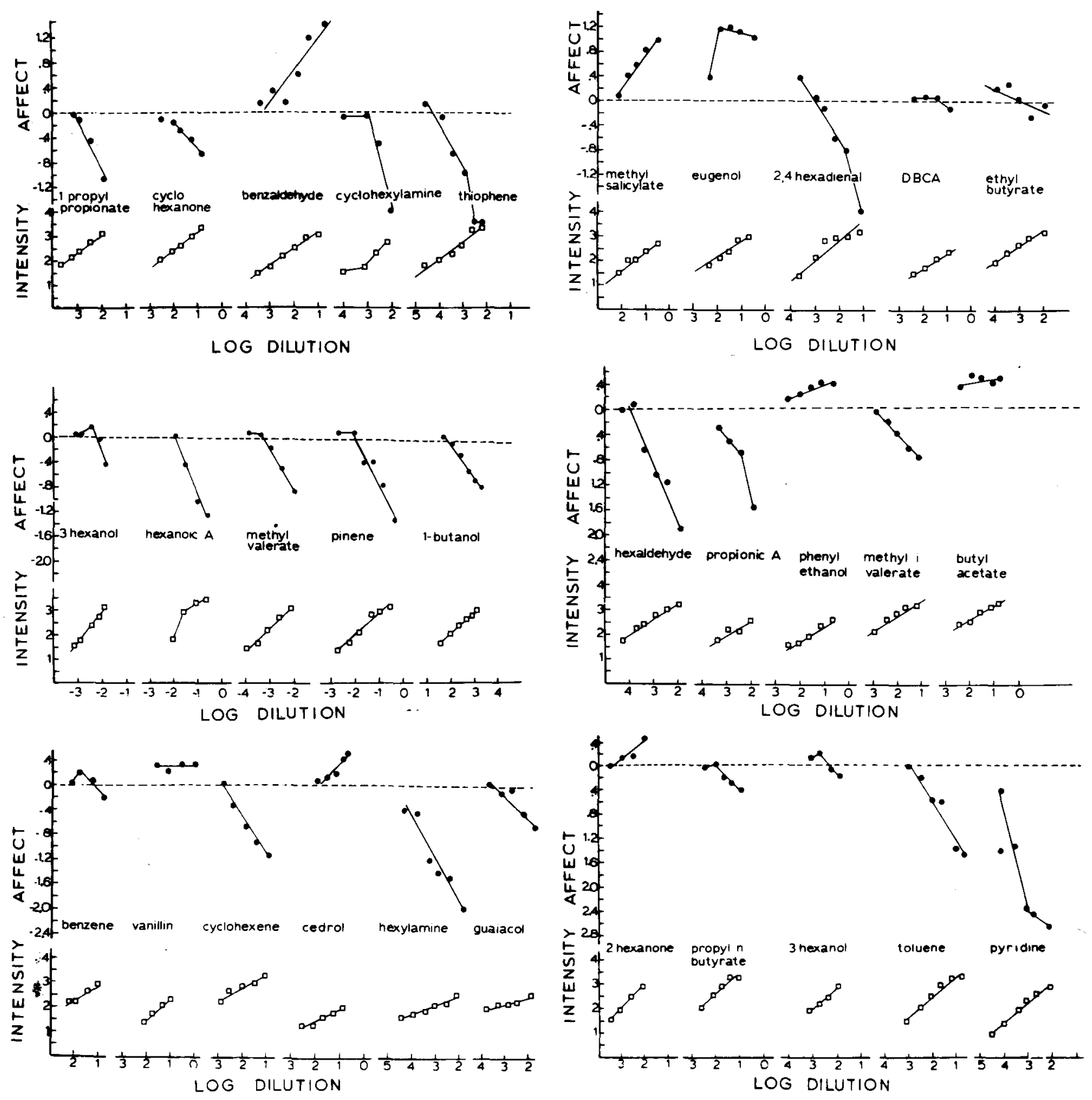

Figure 1. Rebation between the dilution of odorants and both their odor intensity (estimated by a matching level of butanol) and their pleasantness (pleasant, unpleasant). The concentration of the odorants is expressed as log dilution, with the scale reading backwards. The intensity of the odorant is given in $\log$ parts per million of butanol judged, on the average, to be as intense as the odorant. The pleasantness of the odorant (affect) is given in linear terms, with positive numbers reflecting pleasantness and negative numbers reflecting unpleasantness. A dashed line is drawn to show the neutral point.

concentration than is nominally assumed to exist on the basis of dilution level.

\section{Measurement of Relative Intensities}

Occasionally it might prove important to estimate the relative odor intensities of a variety of chemicals. The present findings suggest that the slopes of the odor intensity functions vary over more than a $3: 1$ range. In log-log coordinates, this means that the functions are separated by varying distances across the concentration scale, and that no single distance (or ratio in linear values) suffices to express the relative odor intensity of one odor compared to another. A compromise solution is to compare the magnitude estimates of two odorants at a single, fixed concentration (e.g., 100\% saturation) by computing the value of their psychophysical functions at that concentration (even though neither odorant had ever 
been directly tested at that concentration). The values of $k_{1}$ in Table 1 provide this estimate for the different odorants. The other solution is much more tedious, for it involves computing the relative odor intensities at each concentration desired.

Pleasantness functions. Figure 1 also shows the arithmetic mean preference ratings across observers, taking into account judgments of liking $(+)$, disliking $(-)$, and neutrality (0). Although many of the mean ratings were slightly above or slightly below 0 , suggesting a definite liking or disliking, median ratings of these concentrations are often 0 .

Two distinct functions appear in Figure 1. One type is a monotonic increase in pleasantness (or unpleasantness) with concentration, which suggests that odor hedonics is highly correlated with odor intensity. The other type is functions that exhibit a maximum pleasantness value at some intermediate concentration, and then become less pleasant (and finally unpleasant) with increasing concentration. A century ago, Wundt (see Beebe-Center, 1932) suggested that all stimuli conform, in their hedonic functions, to an inverted-U-shaped curve. Low levels are pleasant, intermediate levels reach the maximum pleasantness, whereas high levels diminish in pleasantness. The second type of odorant (e.g., hexaldehyde, 3 hexanol, eugenol, ethyl butyrate, propyl $n$ butyrate, benzene) confirm Wundt's speculation. The first type of odorants do not, perhaps because the odorants tested here were chosen at one region of concentration, inappropriate for the breakpoint to occur.

Table 1 presents the parameters of the three parameter power functions relating odor pleasantness to percent concentration. In some cases, the functions provide an excellent fit. In others, in which there are sharp angular shifts in the function, the power functions provide only a modest fit. A visual inspection of the functions suggests that there is a fair symmetry between the pleasantness portions of a function and its unpleasant portion (for those cases in which a function exhibits both a pleasantness and an unpleasantness range). In contrast, previous studies evaluating a variety of tastants suggested that pleasantness and unpleasantness are governed by two different laws-pleasantness functions (e.g., for sweetness) are usually flatter (against stimulus concentration) than unpleasantness functions (Moskowitz et al., 1975).

In a previous tudy (Moskowitz, Kluter, Westerling, \& Jacobs, 1974), the suggestion was made that for taste stimuli (especially sweetness) the optimal level occurred at a fixed sweetness level (i.e., at a fixed psychological magnitude). After a certain perceived sweetness is reached, the observer no longer finds the increments in concentration to add any additional palatability to the stimulus. Do there exist any such invariant points on the pleasantness function for odor stimuli? That is, does the point at which the odorant becomes neutral (or maximizes in pleasantness) conform to a constant dilution level, or even more desirable, a constant perceived intensity level. Pleasantness functions obtained here usually go through neutrality at concentrations ranging between 1.5 and $2.5 \log$ PPM of butanol. This is a one-log-unit range, corresponding to a $10^{0.66}=4.57$ range of intensities. Since the psychological range of odor intensities is small, one may assume that for odor stimuli the neutrality region does not occur at a fixed odorant intensity, but varies idiosyncratically with the odorant.

\section{Inverse Relations Between Intensity and Pleasantness}

Moskowitz, Dravnieks, and Gerbers (1974) suggested that for l-butanol odor intensity is the inverse of odor pleasantness when group data are considered, although the inverse relation may break down when data from individual observers are analyzed. Henion (1971) had suggested the same inverse relation for the banana-like odorant, amyl acetate. In both studies, observers were required to use positive numbers to represent both intensity and pleasantness (or unpleasantness). Here the observers are allowed to use positive and negative numbers to reflect graded degrees of liking and disliking. The average ratings for pleasantness can be plotted against the average ratings for intensity. In order to determine whether the relation is truly inverse, at the group level, the following steps were taken: (a) The arithmetic mean pleasantness (or unpleasantness) rating was computed by averaging together positive and negative ratings. (b) For those odorants in which the preponderance of concentrations were either pleasant or unpleasant, the mean ratings were plotted as a function of odor intensity in log-log coordinates. The absolute values of the average pleasantness ratings were thus used. (c) Aberrant concentrations (e.g. those rated pleasant when the remaining concentrations were rated unpleasant) were eliminated.

Figure 2 shows the plots. As a general rule, -odor unpleasantness grows more rapidly than odor intensity. The slopes in Figure 2 are the exponents of power functions of the form: $P=k I^{n}(P=$ hedonic rating, $\mathrm{I}=$ odor intensity). In most cases, $\mathrm{n}$ exceeds 1.0 , suggesting that odor unpleasantness grows more rapidly with concentration than with odor intensity. A word of caution is required, however. The geometric mean intensity rating is plotted on the abscissa, ehreas the arithmetic mean rating is plotted on the ordinate. Typically, the geometric and the arithmetic means differ when all the numbers are positive, so that the actual form of the intensity-pleasantness relation (i.e., the slopes, intercepts) are only approximate in Figure 2. 


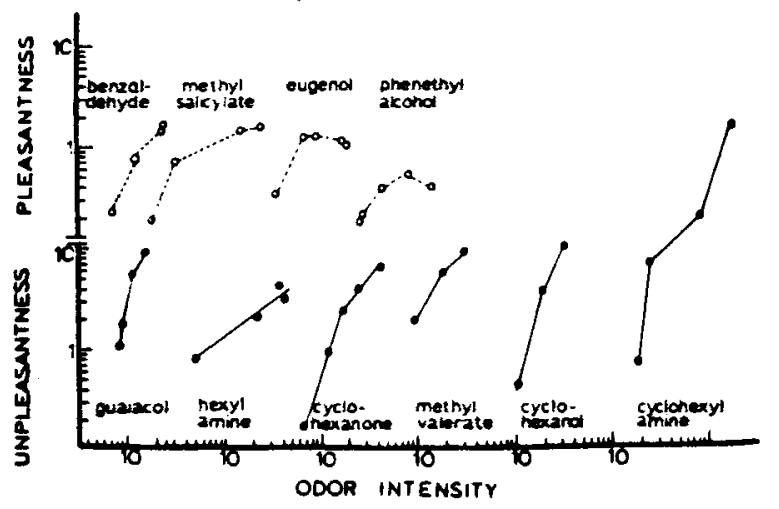

Figure 2. Relation between odor intensity (derived magnitude estimate and arthmetic mean hedonic rating. The coordinates are $\log -\log$, in which straight lines imply functions of the form pleasantness (or unpleasantness) $=\mathbf{k}_{\mathbf{A}}$ (odor intensity) ${ }^{\mathbf{k}} \mathbf{B}$.

As a general rule, the odor pleasantness slopes are steeper than the odor intensity slopes for those odorants showing a preponderance of either positive or negative values. Similar dominance of pleasantness over intensity slopes appeared for the data of Moskowitz, Dravnieks, and Gerbers (1974), although to a smaller degree than found here. In addition, the pleasantness functions in Figure 2 are truncated, so that the full hedonic range is missing. The slopes in Figure 2 must be considered as only partial data, and await studies with a more extensive range of concentration.

\section{DISCUSSION}

\section{Size of the Exponent}

Previous scaling exercises have suggested that odor intensity is governed by a power function whose exponent is less than 1.0 . For the variety of qualitatively different odorants investigated here, that supposition is completely borne out if the matches are expressed as power functions, and if the butanol standard function is used to calibrate the equal intensity matches in terms of derived magnitude estimates. Odor intensity belongs to those sensory continua in which the sensory system shrinks the large range of the physical intensities into a much more confined subjective range. Whereas the physical range may vary almost two logarithmic units, the subjective range is limited only to about 20:1.

The previous studies with sniff bottles suggested a much lower power function exponent, with exponent values lying around 0.1-0.2. In many instances, numerous inversions in the intensity functions occurred, so that stimuli of more intense physical level were rated lower than stimuli of lesser intensity. A variety of causes conspire to produce this anomaly. As noted above, the sniff bottle procedure may yield concentrations that nominally have high ranges, but which in actuality are very narrow. Adsorption of the odorant onto the wall of the sniff bottle, poor delivery of the stimuli, and the failure of Raoult's law to work may cause this. Second, because the exponent is so low, fixed errors have a greater tendency to influence the relative position of the mean judgments of adjacent concentrations.

\section{Are There Systematic Relations Between Odor Structure and Exponent?}

The suggestion by Cain (1969) that water-soluble chemicals yield higher exponents than fat-soluable chemicals must be tempered with the realization that a variety of other factors enter into the determination of an exponent. Laffort and Dravnieks (1973) have suggested a variety of physico-chemical factors, and for these they have developed a predictive model for the exponent.

The present study employed too few concentrations of each odorant, and too few replicates to adequately pin down the exponent to more than one decimal place. In many cases, the nonlinearities make the estimation of the exponent difficult. However, one can conclude that odors vary in exponent by a 3:1 range. The highest exponent is for cyclohexanol, a musty smelling, oil-soluble odorant. Its exponent (from the derived magnitude estimation scale) is 0.75 , meaning that its odor intensity is moderately decelerating with concentration. The lowest exponent is guaiacol $(0.21)$, a burnt-smelling odorant which is water and oil soluble.

\section{No Single Exponent Suffices to Characterize All Odorants: Individual Differences}

Individuals differ substantially in what they find pleasing and displeasing in odorants. Part of the variation results from the possibilities open to the observer who can use both positive and negative numbers to express pleasantness and unpleasantness, but who is limited in the use of butanol intensity values to express odor strength. On the other hand, the results of Moskowitz, Dravnieks, and Gerbers (1974) suggest that whereas some observers find an odorant pleasing, other observers may find the same odor equally intense but just as displeasing. The systematic analysis of individual differences may shed light on the processes involved when an individual makes a hedonic judgment of odor, and is a feasible research topic easily implemented by the enterprising investigator.

The assessment of odor pleasantness is much more fraught with difficulties. For many of the odorants evaluated here, the estimates of pleasantness vs. unpleasantness were made without reference to the fact that the stimulus chemical was just a chemical and not a food or other consumable item (e.g., perfume, deodorizer). Hence, this type of situation, in 
Although both the equal intensity matching and the line-matching procedures are less "direct" methods of obtaining estimates of odor intensity and odor pleasantness, respectively, both can be almost immediately transformed into numbers reflecting ratio-scale values of each attribute.

\section{METHOD}

\section{Stimuli and Apparatus}

The stimuli were 32 different, reagent-grade odorants which represented a diversity of odor qualities and were of varying pleasantness and unpleasantness. Their qualities are presented in Table 1. A large number of chemical families are represented, covering the broad spectrum of stimulants in olfaction. The odorants were presented to the observer by means of an eight-channel air-dilution olfactometer, whose details have been previously described (Moskowitz, Dravnieks, \& Gerbers, 1974). Briefly, the olfactometer comprised the following working elements:

(1) Two air sources were used. One passed over the odorant (which was contained in a long glass vessel). The air source saturated itself with the odorant at room temperature, and was led off to a splitter which partitioned the saturated airstream into eight channels. Often predilutions were used, so that a bypass arrangement was set up. $X \%$ of the airstream passed over the butanol. whereas $(100-X) \%$ of the airstream bypassed the saturating tube, and later rejoined the saturated stream before reaching the eight-channel splitter. The second airstream (clean air) entered another splitter, and was split into seven channels.
(2) Eight sniffing ports were available. Each sniffing port received inputs from two airstreams. One was fed at $\mathrm{Y} \mathrm{ml} / \mathrm{min}$ (saturated stream) and the other was fed with $(120-\mathrm{Y}) \mathrm{ml} / \mathrm{min}$ of clean, makeup air. The two tubes leading into the sniffing port met in a wide glas: tube, which mixed the two streams of air and presented them to the nose.

Since the system operated continuously, after approximately $1 / 2 \mathrm{~h}$ of initial operation, the concentrations in all eight ports had reached equilibrium, and so when sniffing each port the observer was presented with a relatively stable concentration. This air dilution olfactometer has the decided advantage over other instruments in that a change in concentration is effected with no re-equilibration time. Even heavy-weight odorants, whick take long times to develop equilibrated concentrations can be tested rapidly and efficiently at a relatively large range of concentration.

\section{Experimental Design and Observers}

Fifteen sessions were run. In each session, between 7 and 10 observers judged between four and six levels of each of two or three odorants. The odorants in any session were selected so that one would be pleasant and the other would be unpleasant. In addition, in one session 1-butanol was tested as both the comparison and the reference odorant. Altogether, 16 observers participated, but some observers ran in more sessions than others.

Each observer first judged the overall odor intensity of each of the four to six test stimuli by sniffing from the port of the olfactometer, and then sniffing from among the eight ports of the reference olfactometer that presented the butanol vapor. The comparison levels of butanol spanned an extremely wide range of sensory

Table 1

Odorants Studied

\begin{tabular}{|c|c|c|c|c|c|c|c|}
\hline Odorant & $\mathbf{k}_{1}$ & $\begin{aligned}= & \mathrm{k}_{1} \\
& \mathrm{k}_{2}\end{aligned}$ & $r^{2}$ & $\mathrm{k}_{3}$ & $\begin{array}{c}P=k_{3} \\
k_{4}\end{array}$ & $\mathrm{k}_{\mathrm{s}}$ & $r^{2}$ \\
\hline Benzene (solvent) & 46.48 & .37 & $.89, .27$ & 13.8 & -.14 & 2.24 & .89 \\
\hline Propyl n butyrate (fruity) & 134.66 & .51 & $.92, .78$ & 6.60 & -18.1 & .41 & .90 \\
\hline L hexanol (alcoholic, metallic) & 149.78 & .65 & $.96, .79$ & 4.15 & -.005 & 3.70 & .98 \\
\hline Hexyalamine (rancid, fishy) & 39.92 & .26 & $.95, .39$ & -514.3 & 322.8 & -.07 & .93 \\
\hline Benzaldehyde (almond, cherry) & 87.99 & .48 & $.96, .75$ & -14.2 & 84.9 & .30 & .90 \\
\hline Eugenol (spicy cloves) & 44.41 & .46 & $.97, .47$ & & & & \\
\hline Hexadienal (grassy) & 138.82 & .45 & $.82, .55$ & 94.4 & -151.8 & .26 & .96 \\
\hline Methyl salicylate (oil of wintergreen) & 24.18 & .46 & $.92, .22$ & -21.7 & 33.4 & .41 & .71 \\
\hline Pinene (turpentine) & 63.95 & .56 & $.98, .79$ & 19.41 & -27.8 & .52 & .95 \\
\hline Vanillin (vanilla) & 15.33 & .35 & $.76, .28$ & & & & \\
\hline Thiophene (rotten, sulfidic) & 320.21 & .46 & $.96, .63$ & 500.61 & -713.60 & .072 & .91 \\
\hline Cedrol (cedar oil) & 9.00 & .26 & $.92, .32$ & -16.2 & 12.5 & .64 & .97 \\
\hline Toluene (solvent) & 93.51 & .50 & $.98, .80$ & 126.7 & -189.3 & .14 & .93 \\
\hline 2 hexanone (sweet, fruity) & 177.03 & .51 & $.95, .65$ & 7.78 & 21.0 & 7.86 & .93 \\
\hline Ethyl butyrate (estery) & 236.29 & .42 & $.96, .65$ & -61.8 & 46.4 & -.14 & .62 \\
\hline 1 propyl propionate (ethereal, estery) & 251.10 & .50 & $.99, .70$ & 9.85 & -86.9 & .85 & .99 \\
\hline Hexanoic acid (fatty, goaty) & 171.73 & .71 & $.89, .68$ & -331.86 & 320.3 & .16 & .98 \\
\hline Cyclohexanol (musty) & 644.23 & .75 & $.96, .68$ & -23.39 & 11.23 & 1.35 & .86 \\
\hline Cyclohexylamine (fishy) & 122.93 & .47 & $.88, .53$ & 6.99 & -173.7 & 1.07 & .99 \\
\hline 3 hexanol (alcoholic) & 164.07 & .51 & $.95, .40$ & 15.09 & -34.2 & .74 & .86 \\
\hline Methyl valerate (harsh, estery) & 427.41 & .58 & $.99, .78$ & 43.57 & -136.2 & .32 & .99 \\
\hline Cyclohexanone (sweet, alcoholic) & 85.36 & .49 & $.99, .48$ & 18.31 & -30.0 & -.35 & .99 \\
\hline Cyclohexene (harsh, solvent) & 89.34 & .34 & $.93, .51$ & -165.67 & 96.6 & -.27 & .99 \\
\hline Butanol (fusel oil) & & & & 182.88 & -195.9 & -.11 & .96 \\
\hline Pyridine (rank, harsh) & 236.29 & .51 & $.95, .60$ & -246.47 & 23.4 & -.54 & .94 \\
\hline Phenylethanol (rose) & 25.69 & 41 & $.98, .53$ & 48.88 & -20.86 & -.45 & .75 \\
\hline Propionic acid (acid, putrid) & 77.92 & .40 & $.89, .30$ & -26.99 & -102.22 & .83 & .99 \\
\hline Dibutylmethyl carbinol acetate (DBCA) (flowery) & 23.46 & .42 & $.99, .40$ & & & & \\
\hline Guaiacol (burnt) & 32.27 & .21 & $.85, .15$ & 11.88 & -88.25 & .48 & .94 \\
\hline Hexaldehyde (grassy, lily) & 225.76 & .42 & $.98, .71$ & 163.27 & -342.58 & .16 & .94 \\
\hline Butyl acetate (banana) & 81.55 & .37 & $.95, .58$ & & & & \\
\hline Methyl iso valerate (estery, harsh) & 110.52 & .38 & $.97, .61$ & 89.80 & -132.1 & .14 & .99 \\
\hline
\end{tabular}

Note-First value in $r^{2}$ column represents averaged data, second represents unaveraged data. 


\begin{tabular}{ccc} 
Comparison of Variability* $\begin{array}{c}\text { Table 2 } \\
\text { for Intensity } \\
\text { Judgments (All Linear Values) }\end{array}$ \\
\hline Concentration & I Hedonic \\
\hline \multicolumn{3}{c}{ Toluene } \\
1 & .49 & H \\
2 & 1.12 & 5.45 \\
3 & 3.66 & 10.60 \\
4 & 2.09 & 26.92 \\
5 & 2.62 & 25.84 \\
6 & 2.82 & 35.97 \\
& Thiophene & 34.17 \\
1 & .76 & 31.58 \\
2 & 1.53 & 27.79 \\
3 & 2.81 & 24.93 \\
4 & 3.53 & 29.52 \\
5 & 2.91 & 36.26 \\
6 & 4.30 & 40.05 \\
& Benzaldehyde & \\
1 & .52 & 21.00 \\
2 & .50 & 22.55 \\
3 & 1.86 & 13.73 \\
4 & 2.97 & 24.64 \\
5 & 2.78 & 43.06 \\
6 & 2.46 & 35.47 \\
\hline
\end{tabular}

*Derived magnitude estimate

which observers judge pure chemicals, is ambiguous for the observer. The preference cues are usually lacking-observers must rely on innate liking and disliking. without the other cues that usually accompany the stimulus. As a result, it should not seem surprising that for most odorants there is a great deal of variability in the judgments. This applies to predominantly pleasant odors, neutral ones (on the average), and slightly unpleasant ones. Definitely unpleasant odorants like pyridine and thiophene are characterized by less variability in subjective judgments of their unpleasantness.

Although most of the odorants here were judged to be in the same range of intensities, there is some suggestion that unpleasant odors like thiophene are judged at high intensities to be substantially more intense than other odorants at their respective highest levels. Part of this may result from thiophene being that intense at high concentrations. On the other hand, there seems to be a confusion between very unpleasant odors and very strong ones, reminiscent of a finding by Moskowitz and Gerbers (1974). They selected 15 odorants to be of the same odor intensity, and instructed observers to estimate (by magnitude estimation) the intensity, the pleasantness, and a variety of quality attributes. The two rankest odorants, methyl disulfide and caproic acid, were judged to be more intense than the other odorants, even though these two had originally been matched ahead of time. Methyl disulfide and caproic acid were also judged to increase in odor intensity from one test day to the next, by the same observers.

\section{Are Intensity and Pleasantness the Same Continuum?}

The present data strongly suggest that, although odor intensity and odor pleasantness may be correlated, observers can and do separate the two attributes. On the other hand, odor hedonics may be sufficiently pervasive to cloud the judgment of odor intensity (e.g., as in the case of methyl disulfide). Substantially further work is needed to disentable the two dimensions. Paradigms in which the observer is repeatedly exposed to an odorant to reduce its hedonic impact may reveal systematic shifts in the function for odor hedonics, and relatively little shift in the function for odor intensity. Such an experimental manipulation is possible with repeated presentations (Moskowitz \& Gerbers, 1974) and recommends itself as a logical next step in the study of odor pleasantness.

\section{REFERENCES}

Beebe-Center, J. G. The psychology of pleasantness and unpleasantness. New York: Van Nostrand, 1932.

Berglund, B., Berglund, U., Ekman, G., \& Engen, T. Individual psychophysical functions for 28 odorants. Perception \& Psychophysics, 1971, 9, 379-384.

CaIN, W. S. Odor intensity: Differences in the exponent of the psychophysical function. Perception \& Psychophysics, 1969, 6. 349-354.

Cain, W. S., \& Moskowitz, H. R. Psychophysical scaling of odors. In J. W. Johnston, D. Moulton, \& A. Turk (Eds.). Human responses to environmental odors. New York: Academic Press, 1974. Pp. 2-32.

ENGEN. T.. \& MCBURney, D. H. Magnitude and category scales of the pleasantness of odors. Joumal of Experimental Psychology. $1964,68,435-440$.

Henion, K. E. Odor pleasantness and intensity: A single dimension? Journal of Experimental Psychology, 1971, 90. 275-279.

Laffort. P..\& Dravnieks. A. An approach to a physico-chemical model of olfactory stimulation in vertebrates by single compounds. Joumal of Theoretical Biology, 1973, 38, 335-345.

Moskowitz, H. R. The sweetness and pleasantness of sugars. American Journal of Psychology, 1971, 84. 387-405.

Moskowitz. H. R., Dravnieks, A., Cain, W. S., \& Turk, A. Standardized procedure for expressing odor intensity. Chemical Senses and Flavor, 1974, 1, 235-237.

Moskowitz, H. R., Dravnieks, A,, \& Gerbers, C. Odor intensity and pleasantness of butanol. Journal of Experimental Psychology, 1974, 103, 216-223.

Moskowitz, H. R., \& Gerkers, C. Dimensional salience of odors. Annals of the New York Academy of Sciences (Odors: Evaluation, Utilization and Control), 1974, 237, 3-16.

Moskowitz, H. R., Kluter, R. A., Westerling, J., \& Jacobs, H. L. Sugar sweetness and preference: Evidence for different psychological laws. Science, 1974, 184, 583-585.

Moskowitz, H. R., Sharma, K. N., Jacoes, H. L., Sharma, S. D., \& Kumrainh, V. Glucose load, but not meals, alters sugar palatability. Physiology and Behavior, 1976, in press.

Peryam, D. R., \& Pilgrim, F. J. Hedonic scale method for measuring food preference. Food Technology, 1957, 9, 11-14.

Stevens, J. C. A comparison of ratio scales for the loudness of white noise and the brightness of white light. Doctoral dissertation, Harvard University, 1957.

Yoshida, M. Studies of psychometric classification of odors (4 and 5). Japanese Psychological Research. 1964, 6, 115-124, 145-154.

(Received for publication March 20, 1975; revision received October $16,1975$. ) 